\title{
Periodontitis Apical: Caracterización Histológica y Morfométrica de Quistes Radiculares y Granulomas Periapicales
}

\author{
Apical Periodontitis: Histological and Morphometric Characterization \\ of Radicular Cysts and Periapical Granulomas
}

Ramón Fuentes $^{1,2}$; Giannina Álvarez ${ }^{2,3}$; Alain Arias ${ }^{1,2,4}$; Evelyn Borie-Echevarría ${ }^{5}$ \& Fernando Dias ${ }^{1,2}$

FUENTES, R.; ALVAREZ, G.; ARIAS, A.; BORIE-ECHEVERRÍA, E. \& DIAS, F. Periodontitis apical: caracterización histológica y morfométrica de quistes radiculares y granulomas periapicales. Int. J. Morphol., 36(4):1268-1274, 2018.

RESUMEN: La periodontitis apical es una patología inflamatoria que afecta los tejidos periapicales de un diente desvitalizado. El objetivo de este estudio fue caracterizar histológica y morfométricamente las lesiones de quistes y granulomas utilizando microscopía óptica. Se analizaron seis biopsias obtenidas de dientes con indicación de exodoncia. El análisis histológico se realizó mediante microscopía óptica y microfotografías, con análisis de contraste de imágenes y conteo celular mediante ImageJ. Descripción de las características histológicas: en los quistes se observaron cavidades rodeadas de epitelio escamoso estratificado no queratinizado y una cápsula fibrosa compuesta de fibrocitos, fibroblastos e infiltrado inflamatorio crónico; en los granulomas se observaron capilares, una capsula fibrosa de fibrocitos/fibroblastos y un infiltrado inflamatorio de predominio linfocitario. Cuantificación del número celular de infiltrado inflamatorio: para quistes fue de $9,2 \mathrm{cel} / 10000 \mu \mathrm{m}^{2}$, mientras que para granulomas fue de $20,8 \mathrm{cel} / 10000 \mu \mathrm{m}^{2}$, sin diferencias estadísticas significativas entre ambos $(\mathrm{p}=0,654)$. Cuantificación del número celular de fibrocitos/fibroblastos: para quistes fue de $15,4 \mathrm{cel} / 10000 \mu \mathrm{m}^{2}$, mientras que para granulomas fue de $18,5 \mathrm{cel} / 10000 \mu \mathrm{m}^{2}$, sin diferencia estadística significativa ( $\left.\mathrm{p}=0,499\right)$. Porcentaje de colágeno tipo I: para los quistes fue de $37,8 \pm 19,2 \%$, mientras que para granulomas fue de $33,8 \pm 23,3 \%$, sin diferencias estadísticas significativas $(\mathrm{p}=0,704)$. Se observó una correlación negativa moderada para el infiltrado inflamatorio $(\mathrm{R}=0,637)$ y una correlación positiva baja para fibrocitos/fibroblastos ( $\mathrm{R}=0,121)$, en relación a la cantidad de colágeno tipo I. Medición del área de las lesiones periapicales: el promedio total de las lesiones fue de 10,7 $\pm 5,0 \mathrm{~mm}^{2}$, siendo el mayor tamaño un quiste de $18,1 \mathrm{~mm}^{2}$ y el menor un granuloma de $5,2 \mathrm{~mm}$. El análisis histológico permite realizar un diagnóstico diferencial de lesiones con características similares y así definir el tratamiento más adecuado.

PALABRAS CLAVE: enfermedad periapical; quiste; granuloma; histopatología.

\section{INTRODUCCIÓN}

La periodontitis apical consiste en el establecimiento de un proceso inflamatorio localizado en los tejidos periapicales, como consecuencia de la infección y posterior desvitalización del diente (Zero et al., 2011; Zohrabian \& Abrahams, 2015). La respuesta del huésped a la persistencia del estímulo microbiano se traduce en una lucha constante por la defensa y mantención del equilibrio mediante procesos de inflamación, reabsorción y destrucción tisular, con la subsecuente generación de una lesión osteolítica periapical, posible de visualizar radiográficamente y de identificar histológicamente como granuloma periapical o quiste radicular (Nair, 2004; Schulz et al., 2009; Zero et al.). Los quistes radiculares son las lesiones quísticas de origen inflamatorio más comunes en los maxilares, estando ubica- das cerca y alrededor de los ápices de los dientes afectados y generalmente precedidos por un granuloma periapical. Este último consiste en la formación de tejido de granulación con un infiltrado celular y una cápsula de tejido conectivo (Nair; Carrillo et al., 2008).

De acuerdo a la clasificación de tumores de cabeza y cuello de la Organización Mundial de la Salud (OMS), los quistes radiculares se encuentran dentro de la entidad de quistes odontogénicos de origen inflamatorio (Speight $\&$ Takata, 2018). Respecto de la etiopatiogenia de los quistes periapicales se ha propuesto que estos se forman a partir de la estimulación de los restos epiteliales de Malassez en el ligamento periodontal, provenientes de la degrada-

\footnotetext{
${ }^{1}$ Departamento de Odontología Integral Adultos, Facultad de Odontología, Universidad de La Frontera, Temuco, Chile.

${ }^{2}$ Centro de Investigación en Ciencias Odontológicas (CICO), Facultad de Odontología, Universidad de La Frontera, Temuco, Chile.

${ }^{3}$ Programa de Magíster en Odontología, Facultad de Odontología, Universidad de La Frontera, Temuco, Chile.

${ }^{4}$ Universidad Adventista de Chile, Chillán, Chile.

${ }^{5}$ Facultad de Ciencias, Universidad Mayor, Chile.
} 
ción de la vaina de Hertwig durante la odontogénesis, mediante los mecanismos y productos involucrados en la inflamación periapical (Nair; Lin et al., 2007; Carrillo et $a l$.). Histológicamente se mencionan dos tipos de quistes, los quistes verdaderos, que se encuentran completamente rodeados de epitelio, y los quistes de bolsillo, que se caracterizan por su cavidad abierta hacia el conducto radicular (Simon, 1980; Ramachandran Nair et al., 1996; Nair). Los quistes radiculares se presentan revestidos en forma parcial o total por un epitelio escamoso estratificado no queratinizado, cuyas células se encuentran unidas por desmosomas (Simon; Ramachandran Nair et al.; Schulz et al.). La pared fibrosa de tejido conectivo presenta diferentes grados de infiltrado inflamatorio celular, compuesta principalmente por macrófagos, neutrófilos, células plasmáticas, linfocitos, además de vasos sanguíneos, cristales de colesterol, cuerpos de Rushton, depósitos de hemosiderina y material de cuerpo extraño (Schulz et al.; Chi \& Neville, 2011). Los granulomas periapicales, en tanto, se caracterizan histológicamente por presentar un infiltrado celular inflamatorio crónico con linfocitos $\mathrm{T}$ y $\mathrm{B}$, células plasmáticas y neutrófilos, una cápsula fibrosa con algunos fibroblastos, vasos sanguíneos truncados y podría encontrarse material de cuerpo extraño, pudiendo presentar o no zonas con epitelio (Ramachandran Nair et al.). La incidencia de estas lesiones es variable, reportándose para el caso de los quistes radiculares entre un $8,6 \%$ y 64,91 $\%$, mientras que para los granulomas periapicales entre un $20 \%$ y $72 \%$ (Ramachandran Nair et al.; Nair; Carrillo et al.; Schulz et al.; Diegues et al., 2011; Akinyamoju et al., 2014; Çalıs san et al., 2016; Sullivan, et al., 2016; Tavares et al., 2017).

En la identificación y diagnóstico de las lesiones periapicales, se han señalado varios métodos de comprobada eficacia, siendo la radiografía periapical la más utilizada (de Paula-Silva et al., 2009). Sin embargo, para el diagnóstico diferencial de estas lesiones, el análisis radiográfico por sí sólo no es concluyente debido a diversos factores que dificultan el diagnóstico, entre ellos, las distintas lesiones osteolíticas de características similares, variabilidad en las características de granulomas y quistes radiculares, y limitaciones de visualización radiográfica bidimensional (Cotti et al., 1999; Lia et al., 2004; de PaulaSilva et al.). Çalıs kan et al. señalan que para llegar a una confirmación diagnóstica definitiva, es necesario el análisis histopatológico de las biopsias de estas lesiones y la correlación con los hallazgos clínicos y radiográficos (Ricucci \& Bergenholtz, 2004; García et al., 2007). El objetivo de este estudio fue presentar una caracterización histológica y morfométrica de biopsias de lesiones periapicales relacionadas a dientes desvitalizados en diferentes pacientes, mediante microscopía óptica.

\section{MATERIAL Y MÉTODO}

Se analizaron seis biopsias de lesiones periapicales obtenidas a partir de dientes con indicación de exodoncia y que en la radiografía preoperatoria presentaban lesiones compatibles con quistes y granulomas. La indicación de exodoncia, formaba parte del tratamiento protésico rehabilitador programado en la consulta privada para cada paciente. Posterior a la exodoncia y biopsia de las lesiones, los pacientes fueron tratados con regeneración ósea guiada e implantes. Cada paciente firmó un consentimiento informado al inicio de su tratamiento. El diagnóstico de periodontitis apical se realizó mediante la anamnesis, examen clínico y radiográfico, lo cual fue consignado en la ficha clínica de cada paciente. La confirmación diagnóstica se realizó mediante análisis histopatológico de las biopsias en el Laboratorio del Centro de Excelencia en Estudios Morfológicos y Quirúrgicos (CEMyQ) de la Universidad de la Frontera.

Procesamiento y análisis de las muestras. Posterior a la exodoncia de los dientes y biopsia de las lesiones apicales, las muestras de tejido fueron fijadas con formalina tamponada al $10 \%$ y almacenadas a $4{ }^{\circ} \mathrm{C}$ por cuatro semanas y lavadas con suero fisiológico. Luego de la inclusión en bloques de parafina, se utilizó un micrótomo Leica ${ }^{\circledR}$ RM2255 para el corte longitudinal de las muestras con $3 \mu \mathrm{m}$ de grosor. Se realizó el procedimiento de rutina para tinciones de Hematoxilina Eosina (HE) y Picrosirius Red. Posteriormente, las muestras fueron analizadas con microscopio óptico Leica ${ }^{\circledR}$ DM750 y cámara Leica ${ }^{\circledR I C C} 50$ HD, a magnificaciones de 10x y 40x.

Área de la lesión periapical. El área aproximada de la lesión periapical se determinó mediante la utilización del software ImageJ (National Institutes of Health, Bethesda, MD). Se trabajó con la imagen completa de la muestra en tinción HE y magnificación de 10x. Se modificaron los contrastes con el software y se trabajó con la imagen en color binario (blanco y negro) para calcular el porcentaje de área seleccionada y realizar posteriormente la conversión a mm2. La obtención del área de la muestra, se determinó mediante saturación con color negro y posterior cuantificación del número de pixeles de ese color (Fig. 1A), metodología similar a lo presentado en otros estudios cuantitativos histológicos (Ziago et al., 2017).

Densidad celular. Para cada uno de los casos, se seleccionaron en forma aleatoria seis segmentos de la muestra con tinción HE a una magnificación de 40x. Una vez seleccionados los segmentos, se realizó una nueva aleatorización y se seleccionaron tres áreas por cada uno. De esta manera se realizó una cuantificación celular por área en forma manual utilizando ImageJ (Fig. 1B). Se contabilizaron dos categorías de células: infiltrado inflamatorio y células de tejido conectivo, sin 
A

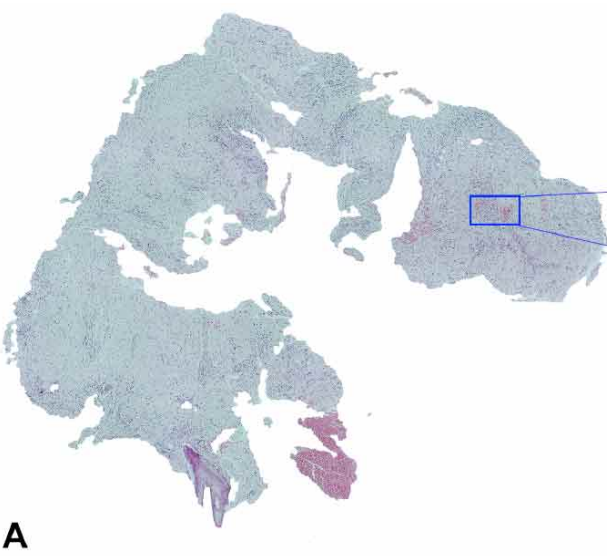

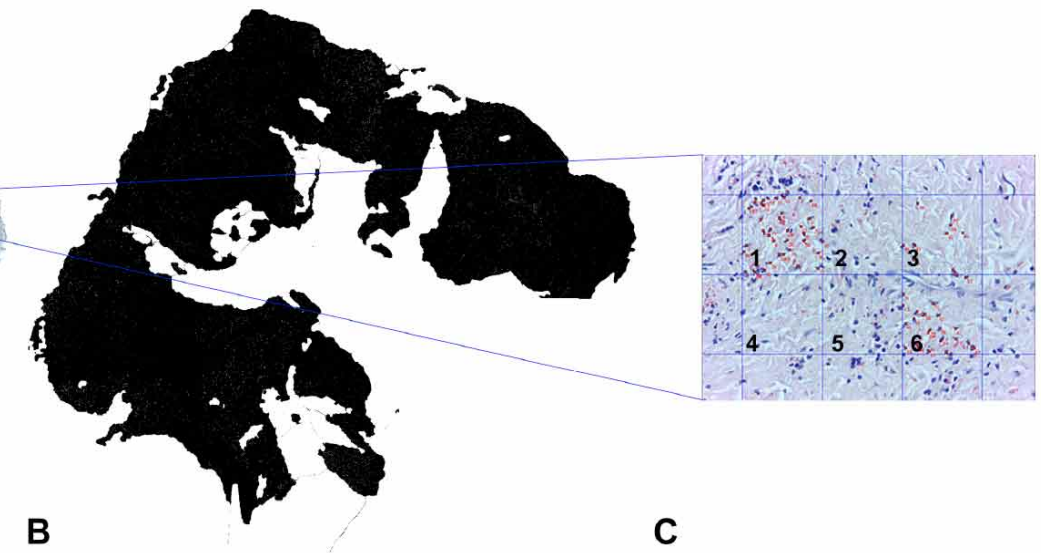

Fig. 1. Metodología de análisis histológico. A) Selección del área a calcular de la imagen original (10x). B) Imagen modificada a color binario para calcular el tamaño o extensión de la lesión en $\mathrm{mm}^{2}$ (área color negro). C) Magnificación de una de las selecciones de la muestra. Se realizó una segunda selección al azar de tres segmentos centrales (1, 3 y 6) para realizar la contabilización de las células presentes. En la imagen se observa predominio de linfocitos y fibrocitos. Tinción de HE con magnificación de 40x
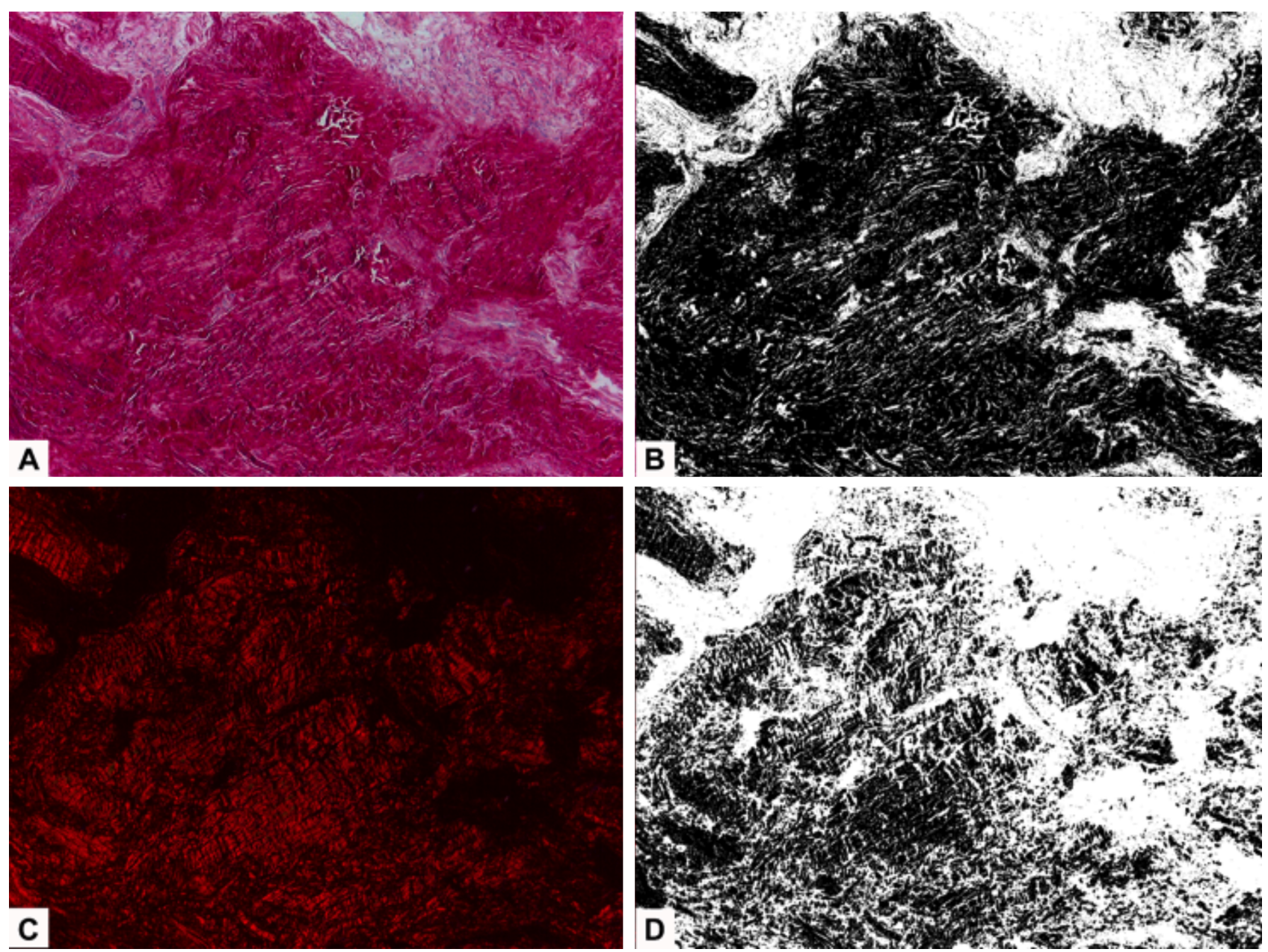

Fig. 2. A) Imagen original con tinción Picrosirius Red, magnificación de 10x. B) Cambio de la imagen a color binario que muestra el total de colágeno en el tejido (color negro). C). Polarización de la imagen original (A) que muestra la cantidad de colágeno tipo I. D) Cambio de la imagen polarizada (C) a color binario para el cálculo del porcentaje de colágeno tipo I (color negro). 
discriminación en el tipo celular. Para el infiltrado inflamatorio se contabilizaron linfocitos, macrófagos, neutrófilos y células plasmáticas, mientras que para las células del tejido conectivo se contabilizaron fibrocitos y fibroblastos.

Densidad de Colágeno Tipo I. Se seleccionaron aleatoriamente tres segmentos de cada muestra con tinción Picrosirius Red. Con el software ImageJ, se modificó el contraste de las imágenes y se trabajó en color binario (blanco y negro). Con ello se calculó el porcentaje de área seleccionada (negro) que corresponde al colágeno total. Posteriormente se realizó el mismo procedimiento, pero con imágenes obtenidas a partir de luz polarizada. De esta forma se contabilizó el porcentaje de área correspondiente a colágeno tipo I. Con los valores de colágeno total y colágeno tipo I, se calculó una proporción o porcentaje. El proceso de modificación del color se presenta en la Figura 2.

Análisis estadístico. Para el análisis estadístico se utilizó el software SigmaPlot 12.0 (Systat Software, Inc, San Jose, CA, USA). Se utilizó la prueba Shapiro-Wilk para determinar la normalidad de los datos. Los datos con distribución normal fueron presentados con la media y desviación, y se aplicó la prueba T. Los datos con distribución no normal fueron presentados con la mediana y cuartiles 1 y 3 , y se aplicó la prueba U Mann Whitney. Se utilizó como umbral de significancia $\mathrm{p}<0,05$.

\section{RESULTADOS}

Aspectos generales. La media de las edades de los pacientes fue de 56,2+10 años. El 33,3\% de las lesiones fueron obtenidas de la zona maxilar y el $66,7 \%$ de la zona mandibular, siendo la zona anterior más frecuente $(66,7 \%)$. Tal como se muestra en la Tabla I, de las biopsias analizadas el $66,7 \%(n=4)$ correspondieron a quistes radiculares y el $33,3 \%(n=2)$ a granulomas. El área promedio de las lesiones periapicales fue de $10,7 \pm 5 \mathrm{~mm}^{2}$, siendo la lesión de mayor tamaño un quiste de $18,1 \mathrm{~mm}^{2}$ y la lesión de menor tamaño un granuloma de $5,2 \mathrm{~mm}^{2}$

Densidad de celular. De acuerdo al análisis y contabilización de la densidad celular inflamatoria, no hubo diferencia estadística entre quistes y granulomas (Fig. 3; p=0,654). La mediana de células inflamatorias en quistes fue de 9,2 cel/ $10000 \mu \mathrm{m}^{2}$ (Q1-1,5; Q3-33,5), mientras que para los granulomas fue de 20,8 cel/10000 $\mu^{2}$ (Q1-0,4; Q3-47,7). En cuanto a la densidad de fibrocitos/fibroblastos presentes en quistes y granulomas, tampoco se encontraron diferencias estadísticas significativas $(\mathrm{p}=0,499)$. En los quistes, la mediana de densidad celular fue de $15,4 \mathrm{cel} / 10000 \mu \mathrm{m}^{2}(\mathrm{Q} 1-$ 9,2; Q3-24,2), mientras que para los granulomas fue de 18,5 cel/10000 $\mu \mathrm{m}^{2}$ (Q1-11,2; Q3- 24,2).

Tabla I. Antecedentes generales de las biopsias y área de las de lesiones periapicales en $\mathrm{mm}^{2}$.

\begin{tabular}{cccccc}
\hline $\mathrm{N}^{\circ}$ de biopsia & Tipo de lesión & Sexo & Edad (años) & Diente & Área de la lesión $\left(\mathrm{mm}^{2}\right)$ \\
\hline 1 & Quiste Radicular & Mujer & 66 & 31 & 10,1 \\
2 & Quiste Radicular & Hombre & 64 & 11 & 18,1 \\
3 & Quiste Radicular & Hombre & 46 & 46 & 11 \\
4 & Quiste Radicular & Hombre & 57 & 41 & 5,6 \\
5 & Granuloma & Mujer & 62 & 46 & 5,2 \\
6 & Granuloma & Hombre & 42 & 22 & 14,3 \\
\hline
\end{tabular}

Densidad de Células Inflamatorias

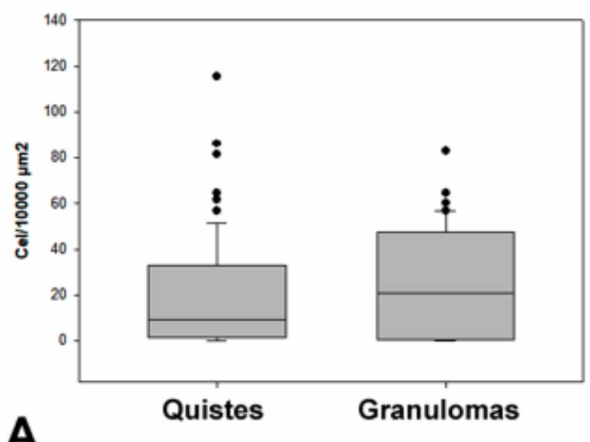

Densidad de Células de Tejido Conectivo

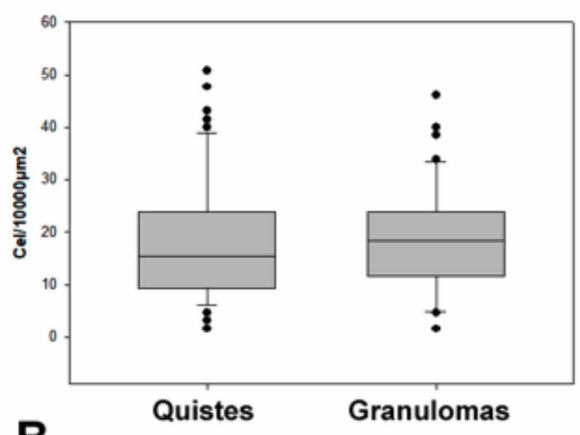

B

Figura 3. A) Densidad celular de células inflamatorias por $10000 \mu \mathrm{m}^{2}$. B) Densidad de células del tejido conectivo (fibrocitos y fibroblastos) por $10000 \mu \mathrm{m}^{2}$. 
Densidad de colágeno tipo I. Respecto a la proporción de colágeno entre quistes y granulomas, no se encontró diferencia estadística significativa (Fig. 4; $\mathrm{p}=0,704$ ). El promedio porcentual de colágeno tipo I para los quistes fue de $37,8 \pm 19,2 \%$, mientras que para los granulomas fue de $33,8 \pm 23,3 \%$.

Correlación entre la densidad celular y cantidad de colágeno tipo I. Se observó una correlación negativa moderada entre la cantidad de células inflamatorias y el porcentaje de colágeno tipo I presente (Fig. 4C; $\mathrm{R}=0,637$ ). Por otro lado, se observó una correlación positiva baja entre la densidad de fibroblastos y el porcentaje de colágeno tipo I presente (Fig. 4D; $R=0,121$ ).

Proporción de Colágeno Tipo I
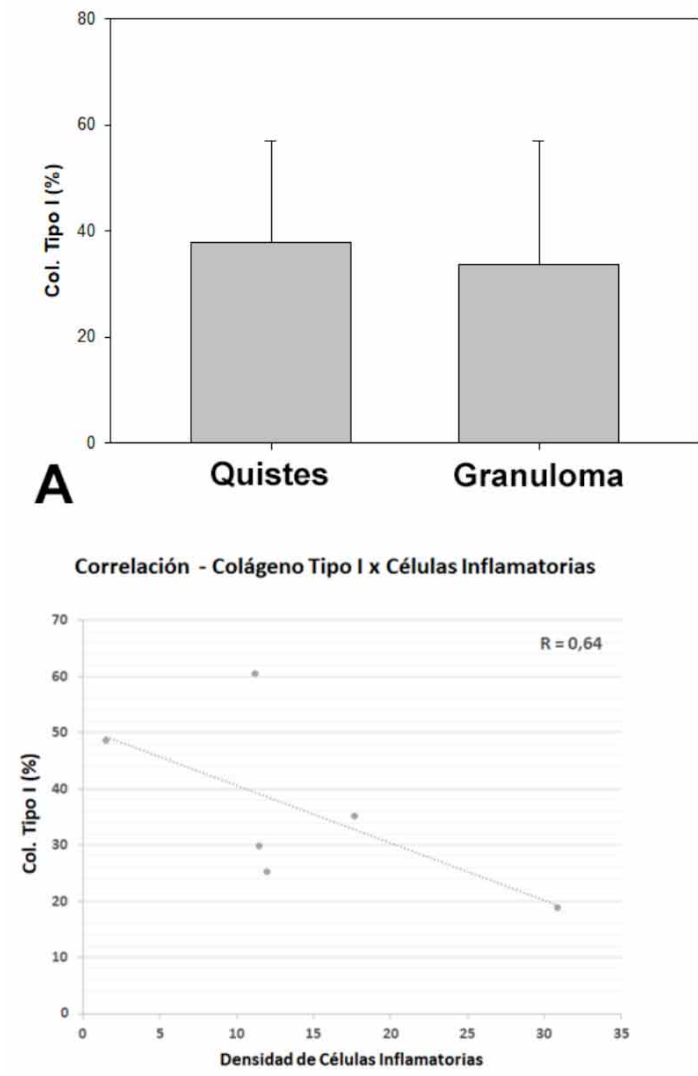

C
Correlación - Colágeno Tipo I x Células Inflamatorias

\section{A}

Densidad de Células Inflamatorias

\section{DISCUSIÓN}

El diagnóstico clínico de periodontitis apical expresado en su forma de quiste radicular o granuloma periapical corresponde en ambos casos a dos etapas distintas del mismo proceso inflamatorio en la patogénesis de la lesión periradicular (Liapatas et al., 2003; Chi \& Neville), por lo que resulta confuso de señalar en ocasiones, que ciertas células puedan estar asociadas exclusivamente o tener mayor predominio con uno u otro tipo de lesión. Liapatas et al. señalaron en su estudio inmunohistoquímico sobre el infiltrado inflamatorio crónico de las lesiones periapicales, que no existen mayores diferencias en el infiltrado inflamatorio ya
Colágeno Tipo I (\%)

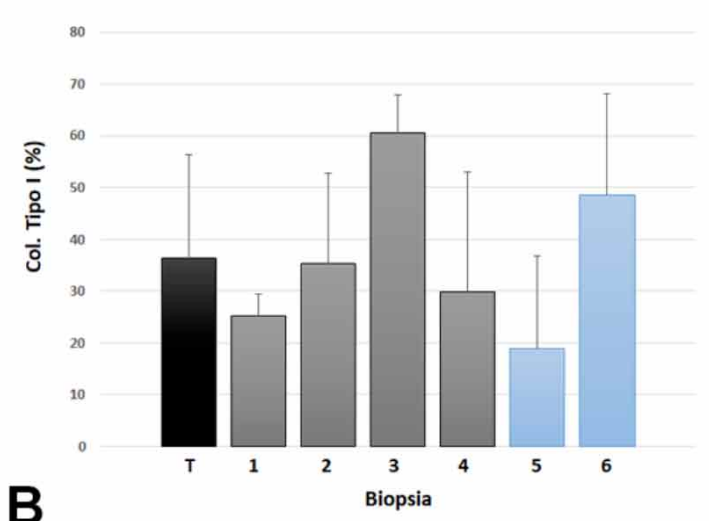

Correlación - Colágeno Tipo I x Células Tejido Conectivo

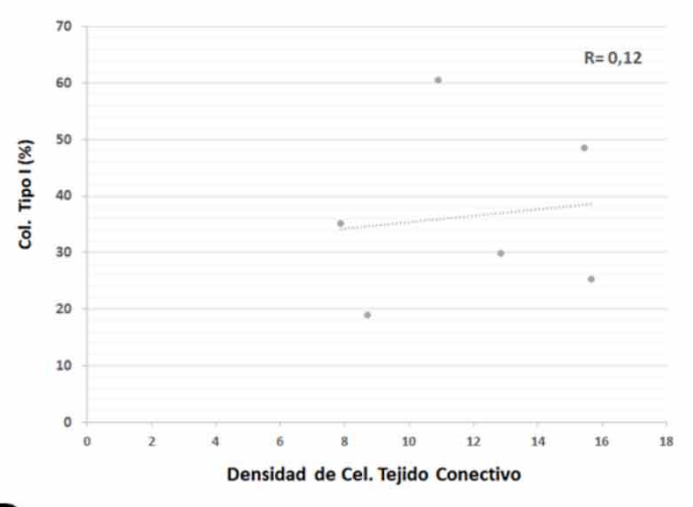

D

Fig. 4. A) Concentración de colágeno tipo I en quistes y granulomas. B) Variación de las concentraciones de colágeno tipo I en las distintas biopsias evaluadas (1-6). La barra de color negro representa el total, las de color gris representan los quistes y las de color azul, los granulomas. C) Concentración de colágeno tipo I (\%) v/s densidad de células inflamatorias $\left(\right.$ cel/10000 $\left.\mu \mathrm{m}^{2}\right)$. D) Concentración de colágeno tipo I (\%) v/s densidad de células de tejido conectivo (cel/10000 $\left.\mu \mathrm{m}^{2}\right)$. 
sea que hablemos de quistes o granulomas. En nuestro estudio tampoco se encontraron mayores diferencias en cuanto a la cantidad de infiltrado inflamatorio tanto para quistes, como para granulomas. Esto muestra de manera general la variabilidad existente entre las distintas lesiones y dentro de la misma lesión, en donde los segmentos tisulares obtenidos no presentaron homogeneidad. Algunos autores agregan que la variabilidad también está relacionada con la procedencia de la muestra, es decir, que los hallazgos histológicos pueden variar si es obtenida por la exodoncia de un diente, como en los casos analizados en este estudio, o por cirugía apical (Schulz et al.).

La incidencia de estas lesiones es muy variable de acuerdo a lo revisado en la literatura. Schulz et al., señalan que esta variabilidad puede estar relacionada con los distintos métodos y clasificaciones para determinar el diagnóstico, la forma de la obtención de la muestra y los métodos de procesamiento de esta (García et al.; Schulz et al.). En este estudio se encontró un mayor número de quistes que granulomas, lo cual coincide con lo reportado por Tavares et al. y Diegues et al., quienes reportan una mayor presencia de quistes que de granulomas en una muestra de población brasileña.

En cuanto al diagnóstico, Çalıskan et al. mencionan que el tamaño radiográfico o la presencia de una línea radiopaca en estas lesiones no son suficientes para establecer un diagnóstico del tipo de lesión y por lo tanto se hace necesario el análisis histopatológico. Lin et al. recomiendan tratar las lesiones de forma inicial con procedimientos conservadores no quirúrgicos, y si se observa persistencia de estas o si el retratamiento endodóntico no quirúrgico no es factible o no está indicado, se recomienda la eliminación de la lesión mediante cirugía apical (Lin et al.; Schulz et al.). Akinyamoju et al. advierten que en muchas ocasiones se produce un sub-diagnóstico, al no realizar el análisis histológico de las lesiones apicales. Así, las ventajas de la biopsia y el correspondiente análisis histológico quedan establecidas en casos en donde lesiones periapicales aparentemente inofensivas resultan ser otras lesiones de mayor gravedad o severidad tales como el adenoma pleomorfo o queratoquiste odontogénico. Kontogiannis et al., y Sullivan et al., en su estudio retrospectivo de lesiones periapicales también encontraron lesiones tumorales como ameloblastoma y queratoquiste odontogénico en el análisis histopatológico, por lo tanto, la diversidad de estas lesiones debe ser considerada y su correcto diagnóstico debe ser priorizado. Considerando que en la práctica clínica el tratamiento en muchas ocasiones se planifica de acuerdo a las manifestaciones clínicas y apoyo de exámenes complementarios imagenológicos, se podría recomendar la realización del análisis histológico de forma rutinaria para mejorar el abordaje terapéutico y ayudar en la comprensión de aquellas lesiones persistentes posterior al tratamiento no quirúrgico.

En nuestro estudio realizamos una propuesta de análisis histológico estructurado y reproducible. Esta metodología de análisis cuantitativo puede proveer información relevante en cuanto a conocer el número de células identificadas por un área determinada. También planteamos una metodología para la cuantificación del colágeno en las lesiones periapicales, que permite hacer una aproximación a la cantidad total de colágeno y distinguir tanto el colágeno tipo I como el tipo III. En este caso, los resultados fueron presentados en relación al colágeno tipo I, con características de fibras densas y gruesas, cuya birrefringencia es intensa entre los colores amarillo y rojo (Junqueira et al., 1978; Vij et al., 2011). Nuestros hallazgos coinciden con los estudios de Mahajan et al. (2013) y Vij et al., que señalan el predominio de estas tonalidades en las paredes de los quistes radiculares, principalmente el color anaranjado.

La periodontitis apical es una patología compleja que requiere dedicación en su diagnóstico para acercarnos a un tratamiento más específico y favorable. En su patogénesis participan diversos factores, en donde tanto los componentes celulares como interacciones moleculares del proceso inflamatorio modifican la progresión y formación de la lesión.

FUENTES, R.; AlVAREZ, G.; ARIAS, A.; BORIEECHEVERRÍA, E. \& DIAS, F. Periodontitis apical: caracterización histológica y morfométrica de quistes radiculares y granulomas periapicales. Int. J. Morphol., 36(4):1268-1274, 2018.

SUMMARY: Apical periodontitis is an inflammatory pathology that affects the periapical tissues of a devitalized tooth. The aim of this study was to histologically and morphometrically characterize lesions of cysts and granulomas using light microscopy. Six biopsies obtained from teeth with indication of exodontia were analyzed. The histological analysis was carried out by means of optical microscopy and microphotographs, with contrast analysis of images and cell count by ImageJ. A description of the histological characteristics was made, observing the cavities surrounded by stratified squamous non-keratinized epithelium and a fibrous capsule composed of fibrocytes, fibroblasts and chronic inflammatory infiltrate; in the granulomas, capillaries, a fibrous capsule of fibrocytes/fibroblasts and a predominantly lymphocytic inflammatory infiltrate were observed. In relation to quantification of the cellular number of inflammatory infiltrate, for cysts itwas of $9.2 \mathrm{cel} / 10000 \mathrm{~mm}^{2}$, while for granulomas it was $20.8 \mathrm{cel} / 10000$ $\mathrm{mm} 2$, without significant statistical differences between both $(\mathrm{p}=$ 0.654 ). The quantification of the fibrocyte / fibroblast cell number was, for cysts, 15.4 cells / $10000 \mathrm{~mm}^{2}$, while for granulomas it was 18.5 cells / $10000 \mathrm{~mm}^{2}$, without significant statistical difference $(\mathrm{p}$ $=0.499$ ). With respect to the percentage of collagen type I, for the cysts was $37.8 \pm 19.2 \%$, while for granulomas it was $33.8 \pm 23.3 \%$, 
without significant statistical differences $(p=0.704)$. A moderate negative correlation was observed for the inflammatory infiltrate $(\mathrm{R}=0.667)$ and a low positive correlation for fibrocytes / fibroblasts $(\mathrm{R}=0.121)$, in relation to the amount of type I collagen. Measurement of the area of the periapical lesions: the total average of lesions were $10.7 \pm 5.0 \mathrm{~mm} 2$, the largest being a cyst of 18.1 $\mathrm{mm} 2$ and the smallest a granuloma of $5.2 \mathrm{~mm} 2$. The histological analysis allows to make a differential diagnosis of lesions with similar characteristics and thus define the most appropriate treatment.

KEY WORDS: Periapical disease; Cyst; Granuloma; Histopathology

\section{REFERENCIAS BIBLIOGRÁFICAS}

Akinyamoju, A. O.; Gbadebo, S. O. \& Adeyemi, B. F. Periapical lesions of the jaws: a review of 104 cases in ibadan. Ann. Ib. Postgrad. Med., 12(2):115-9, 2014.

Çalıskan, M. K.; Kaval, M. E.; Tekin, U. \& Ünal, T. Radiographic and histological evaluation of persistent periapical lesions associated with endodontic failures after apical microsurgery. Int. Endod. J., 49(11):1011-9, 2016.

Carrillo, C.; Peñarrocha, M.; Bagán, J. V. \& Vera, F. Relationship between histological diagnosis and evolution of 70 periapical lesions at 12 months, treated by periapical surgery. J. Oral Maxillofac. Surg., 66(8):1606-9, 2008.

Chi, A. C. \& Neville, B. W. Odontogenic cysts and tumors. Surg. Pathol. Clin., 4(4):1027-91, 2011.

Cotti, E.; Vargiu, P.; Dettori, C. \& Mallarini, G. Computerized tomography in the management and follow-up of extensive periapical lesion. Endod. Dent. Traumatol., 15(4):186-9, 1999.

de Paula-Silva, F. W.; Wu, M. K.; Leonardo, M. R.; da Silva, L. A. \& Wesselink, P. R. Accuracy of periapical radiography and cone-beam computed tomography scans in diagnosing apical periodontitis using histopathological findings as a gold standard. J. Endod., 35(7):1009$12,2009$.

Diegues, L. L.; Colombo Robazza, C. R.; Costa Hanemann, J. A.; Costa Pereira, A. A. \& Silva, C. O. Correlation between clinical and histopathological diagnoses in periapical inflammatory lesions. $J$. Investig. Clin. Dent., 2(3):184-6, 2011.

García, C. C.; Sempere, F. V.; Diago, M. P. \& Bowen, E. M. The postendodontic periapical lesion: histologic and etiopathogenic aspects. Med. Oral Patol. Oral Cir. Bucal, 12(8):E585-90, 2007.

Junqueira, L. C.; Cossermelli, W. \& Brentani, R. Differential staining of collagens type I, II and III by Sirius Red and polarization microscopy. Arch. Histol. Jpn., 41(3):267-74, 1978.

Lia, R. C.; Garcia, J. M.; Sousa-Neto, M. D.; Saquy, P. C.; Marins, R. H. \& Zucollotto, W. G. Clinical, radiographic and histological evaluation of chronic periapical inflammatory lesions. J. Appl. Oral Sci., 12(2):11720, 2004.

Liapatas, S.; Nakou, M. \& Rontogianni, D. Inflammatory infiltrate of chronic periradicular lesions: an immunohistochemical study. Int. Endod. J., 36(7):464-71, 2003.

Lin, L. M.; Huang, G. T. \& Rosenberg, P. A. Proliferation of epithelial cell rests, formation of apical cysts, and regression of apical cysts after periapical wound healing. J. Endod., 33(8):908-16, 2007.

Mahajan, A. M.; Mahajan, M. C.; Ganvir, S. M. \& Hazarey, V. K. The role of stroma in the expansion of odontogenic cysts and adenomatoid odontogenic tumor: A polarized microscopy study. J. Nat. Sci. Biol. Med., 4(2):316-20, 2013
Nair, P. N. Pathogenesis of apical periodontitis and the causes of endodontic failures. Crit. Rev. Oral Biol. Med., 15(6):348-81, 2004.

Ramachandran Nair, P. N.; Pajarola, G. \& Schroeder, H. E. Types and incidence of human periapical lesions obtained with extracted teeth. Oral Surg. Oral Med. Oral Pathol. Oral Radiol. Endod., 81(1):93-102, 1996.

Ricucci, D. \& Bergenholtz, G. Histologic features of apical periodontitis in human biopsies. Endod. Top., 8(1):68-87, 2004.

Schulz, M.; von Arx, T.; Altermatt, H. J. \& Bosshardt, D. Histology of periapical lesions obtained during apical surgery. J. Endod., 35(5):63442, 2009.

Simon, J. H. Incidence of periapical cysts in relation to the root canal. $J$. Endod., 6(11):845-8, 1980.

Speight, P. M. \& Takata, T. New tumour entities in the 4th edition of the World Health Organization Classification of Head and Neck tumours: odontogenic and maxillofacial bone tumours. Virchows Arch., 472(3):331-9, 2018

Sullivan, M.; Gallagher, G. \& Noonan, V. The root of the problem: Occurrence of typical and atypical periapical pathoses. J. Am. Dent. Assoc., 147(8):646-9, 2016.

Tavares, D. P.; Rodrigues, J. T.; Dos Santos, T. C.; Armada, L. \& Pires, F. R. Clinical and radiological analysis of a series of periapical cysts and periapical granulomas diagnosed in a Brazilian population. J. Clin. Exp. Dent., 9(1):e129-35, 2017.

Vij, R.; Vij, H. \& Rao, N. N. Evaluation of collagen in connective tissue walls of odontogenic cysts--a histochemical study. J. Oral Pathol. Med., 40(3):257-62, 2011

Zero, D. T.; Zandona, A. F.; Vail, M. M. \& Spolnik, K. J. Dental caries and pulpal disease. Dent. Clin. North Am., 55(1):29-46, 2011.

Ziago, E. K.; Fazan, V. P.; Iyomasa, M. M.; Sousa, L. G.; Yamauchi, P. Y.; da Silva, E. A.; Borie, E.; Fuentes, R. \& Dias, F. J. Analysis of the variation in low-level laser energy density on the crushed sciatic nerves of rats: a morphological, quantitative, and morphometric study. Lasers Med. Sci., 32(2):369-78, 2017.

Zohrabian, V. M. \& Abrahams, J. J. Inflammatory diseases of the teeth and jaws. Semin. Ultrasound CT MR, 36(5):434-43, 2015.

\section{Dirección para correspondencia}

Prof. Dr. Ramón Fuentes Fernández

Centro de Investigación en Ciencias Odontológicas (CICO)

Facultad de Odontología

Universidad de La Frontera

Av. Francisco Salazar 01145

Temuco

CHILE

E-mail: ramon.fuentes@ufrontera.cl

Recibido : 09-05-2018

Aceptado: 29-07-2018 